Editorial

\title{
Worldwide statins prescription pattern: is it similar?
}

\begin{abstract}
Statins are commonly prescribed to prevent or treat of cardiovascular diseases (CVD) worldwide, preventing about 80,000 stroke and heart attack cases annually. Various statins have been initiated with different biologic properties, chemical structure, safety, efficacy and side effects, with no similar prescription pattern in different countries. One of the most common reasons of the changes among different countries, might be due to the behavior of physicians in various continents.
\end{abstract}

Keywords: statins, prescription, pattern, worldwide, physicians
Volume 9 Issue 6 - 2020

\section{Masoud Amiri}

Department of Epidemiology, Erasmus Medical Center, Rotterdam, The Netherlands

Correspondence: Masoud Amiri, Department of Epidemiology, Erasmus Medical Center, Rotterdam, The Netherlands, Email masoud.amiri@yahoo.com

Received: November II, 2020 | Published: November 27, 2020
Statins are commonly prescribed to prevent or treat of cardiovascular diseases (CVD) worldwide. ${ }^{1}$ A review in The Lancet estimated that statins can annually prevent about 80,000 stroke and heart attack cases. ${ }^{2}$ Lovastatin was the first used statin to lower lowdensity lipoprotein cholesterol (LDL-C) more than four decades ago. ${ }^{3}$ After that, several statins have been initiated with different biologic properties, chemical structure, safety, efficacy and side effects. ${ }^{1}$

The prescription of various statins is not similar in different countries. For example, in Hong Kong, simvastatin, atorvastatin and rosuvastatin were the first three common statins. ${ }^{4}$ Google trends search (from January 2004 to December 2018) has revealed that overall, atorvastatin (39\%) was the most common statin in the world, followed by simvastatin $(26 \%)$, rosuvastatin $(19 \%)$, pravastatin $(8 \%)$, lovastatin $(5 \%)$, fluvastatin $(2 \%)$ and pitavastatin $(1 \%){ }^{3}$ However, the worldwide pattern has been changed from 2016 to 2018, with atorvastatin (44\%), rosuvastatin (22\%), simvastatin (21\%), pravastatin $(8 \%)$, lovastatin $(3 \%)$, pitavastatin $(2 \%)$ and fluvastatin $(1 \%) .^{3}$ In the UK, statins were the most common prescribed medicines in 2016 (about 6 million Britain people took statins to lower the risk of CVD). ${ }^{2}$

In a study on 13 European countries, it has been found that the statin use has been increased about four times from 1997 to $2002 .{ }^{5}$ The drug market varied between countries. For example, Norway had more than five times the per capita statin use compared to Italy; simvastatin and atorvastatin were the most common statins in Europe. ${ }^{5}$

One of the most common reasons of the changes among different countries, might be due to the behavior of physicians in various continents. For example, in a comparison of Honk Kong and USA, it has been found that Hong Kong physicians prefer to prescribe less expensive with lower doses of statins compared to American physicians. ${ }^{6}$ In addition, a study published in 2019, reported that more than 50 percent of patients eligible for statins do not receive them, mainly because their physicians never offered it. ${ }^{7}$

As a conclusion, it seems that despite benefits of statins in preventing CVD, there are still many physicians around the world that do not believe this fact. In addition, there is a huge variation among physicians about the type and dose of statins they prescribe for their patients.

\section{References}

1. Gencer B, Mach F. Lipid management in ACS: Should we go lower faster? Atherosclerosis. 2018;275:368-375.

2. EurActiv. Cardiovasular Health in the UK: Special report; 19-23 December 2016.

3. Lippi G, Mattiuzzi C, Cervellin G. Statins popularity: A global picture. $\mathrm{Br}$ J Clin Pharmacol. 2019;85(7):1614-1615.

4. Blais JE, Chan EW, Law SWY, et al. Trends in statin prescription prevalence, initiation, and dosing: Hong Kong, 2004-2015. Atherosclerosis 2019;280:174-182.

5. Walley T, Folino-Gallo P, Schwabe U, et al. Variations and increase in use of statins across Europe: data from administrative databases. $B M J$. 2004;328(7436):385-386.

6. Wang Y, Nichol MB, Yan BP, et al. Descriptive analysis of real-world medication use pattern of statins and antiplatelet agents among patients with acute coronary syndrome in Hong Kong and the USA. BMJ Open. 2019;9(7):e024937.

7. Bradley CK, Wang TY, Li S, et al. Patient-Reported Reasons for Declining or Discontinuing Statin Therapy: Insights From the PALM Registry. $J$ Am Heart Assoc. 2019;8(7):e011765. 